\title{
LEBESGUE MEASURE OF PARTS FOR $R(X)$
}

\author{
DONALD R. WILKEN ${ }^{1}$
}

1. Introduction. Let $X$ be a compact set in the complex plane $C$. By $R(X)$ is meant the function algebra which consists of functions uniformly approximable on $X$ by rational functions whose poles lie outside $X$. Then $R(X)$ is a subalgebra of $C(X)$, the algebra of all complex valued continuous functions on $X$, and the maximal ideal space of $R(X)$ can be identified with $X$.

In this note we examine the nature of the Gleason parts of $R(X)$ and obtain the result that each nontrivial part has positive planar Lebesgue measure, and each trivial part is a peak point. $\$ 2$ contains a brief summary of the definitions and facts about Gleason parts and representing measures which are relevant to the later proofs. In $\$ 3$ the main result is established along with some noteworthy consequences. The concluding remarks of $\$ 4$ are directed primarily at the numerous unproven but conjectured properties of the parts of $R(X)$.

2. Gleason parts and representing measures. For an arbitrary function algebra $A$ on a compact metrizable space $X$, let $M$ denote its maximal ideal space and $S$ its Silov boundary. Gleason [4] introduced an equivalence relation on $M$ which, realizing $A$ as a function algebra on $M$, can be described as follows:

Definition 2.1. For two points $m_{1}$ and $m_{2}$ in $M, m_{1}$ is (Gleason) equivalent to $m_{2}$ (write $m_{1} \sim m_{2}$ ) if

$$
\sup \left\{\left|f\left(m_{1}\right)\right|: f\left(m_{2}\right)=0,\|f\| \leqq 1\right\}<1 .
$$

Gleason has shown that " " defines an equivalence relation on $M$ and the equivalence classes of $M$ are called the (Gleason) parts of $M$.

Definition 2.2. A representing measure for a point $m$ in $M$ is a positive regular Borel measure $\mu$ on $M$ which satisfies

$$
f(m)=\int f d \mu \text { for all } f \text { in } A .
$$

Each point in $M$ always has a representing measure supported on $S$.

Of special interest to us is the existence of certain representing measures for points which are not peak points for $A$. (A peak point for $A$ is a point in $M$ for which there is a function $f$ in $A$ satisfying $f(m)=1$ and $\left|f\left(m^{\prime}\right)\right|<1$ for $m^{\prime}$ different from $m$ in $M$.) If we let $P$ denote the

Received by the editors June 29, 1966.

1 National Science Foundation Postdoctoral Fellow at Brandeis University. 
set of peak points of $A$, the facts we will need are the following:

Leмma 2.3. If $m$ is not in $P$ there exists a representing measure $\mu$ for $m$ with $\mu(m)=0$.

LEMMA 2.4. Let $\mu_{1}$ and $\mu_{2}$ be representing measures for the points $m_{1}$ and $m_{2}$ in $M$. Let $Q_{1}$ and $Q_{2}$ denote the parts of $M$ containing $m_{1}$ and $m_{2}$ respectively. If $Q_{1} \neq Q_{2}$, then $\mu_{1}$ and $\mu_{2}$ are singular and $\mu_{1}\left(Q_{2}\right)=0=\mu_{2}\left(Q_{1}\right)$.

For a proof of Lemma 2.3 see [2]; for a proof of Lemma 2.4 see [3].

3. $R(X)$. We now return to the case of the algebra $R(X)$. Let $\lambda$ denote Lebesgue planar measure. Then our main result is:

THEOREM 3.1. If a point $x$ in $X$ is not a peak point of $R(X)$, then $Q_{x}$, the part containing $x$, has positive $\lambda$-measure.

We will need the following two lemmas in the proof. Proofs of both of these lemmas are contained in the proof of Theorem 4 of [2].

LemmA 3.2. Let $\nu$ be any positive measure on $X$. Let

$$
N(y)=\int_{X} \frac{d \nu(z)}{|z-y|} .
$$

Then $N(y)$ is finite a.e. $-d \lambda$.

Lemma 3.3. Let $\sigma$ be any measure on $X$. Let

$$
F(y)=\int_{x} \frac{d \sigma(z)}{z-y} .
$$

If $F(y)=0$ a.e. $-d \lambda$, then $\sigma \equiv 0$.

Proof of Theorem 3.1. Let $x$ be as hypothesized. Then Lemma 2.3 provides a representing measure $\mu$ for $x$ such that $\mu$ has no mass at $x$. Let $\delta$ be the unit point mass at $x$. Then both $\mu$ and $\delta$ represent $x$ so that $\mu-\delta=\nu$ is a nonzero measure which annihilates $R(X), \nu \perp R(X)$.

Let $N(y)=\int_{X} d|\nu|(z) /|z-y|$ and $F(y)=\int_{X} d \nu(z) /(z-y)$.

Let $U=\{y \in C: N(y)<\infty$ and $F(y) \neq 0\}$. If $y$ is not in $X$, the function $f(z)=1 /(z-y)$ belongs to $R(X)$. But since $\nu \perp R(X)$ we have

$$
0=\int f(z) d \nu(z)=\int \frac{d \nu(z)}{z-y}=F(y) .
$$

Thus $U \subset X$. Moreover $\lambda(U)>0$. For Lemma 3.2 yields

$$
\lambda(U)=\lambda(\{y \in C: F(y) \neq 0\})
$$

and Lemma 3.3 states 


$$
\lambda(\{y \in C: F(y) \neq 0\})>0 .
$$

To establish the theorem, then, it is enough to show $U \subset Q_{x}$. To do this we utilize Lemma 2.4. Indeed, for each $y$ in $U$ we obtain a representing measure which is absolutely continuous with respect to $\mu$.

Let $f$ be any rational function in $R(x)$. Then

$$
g(z)=(f(z)-f(y)) /(z-y)
$$

is also a rational function in $R(X)$. Hence

$$
0=\int g(z) d \nu=\int \frac{f(z)-f(y)}{z-y} d \nu(z)=\int f(z) \frac{d \nu(z)}{z-y}-f(y) F(y),
$$

since $N(y)<\infty$. But $F(y) \neq 0$ so that

$$
f(y)=\int f(z) \frac{1}{F(y)} \frac{d \nu(z)}{z-y} .
$$

But if the above holds for rational functions in $R(X)$, the same is true for uniform limits, from which we conclude:

$\sigma_{y}=(1 / F(y))(\nu /(z-y))$ is a complex representing measure for $y$ (by which is meant any finite complex Borel measure which satisfies the representing measure equation for $y$ ).

At this poin $t^{2}$ we appeal to a recent result of Hoffman and Rossi [5]. Their theorem states, for arbitrary function algebras, given any complex representing measure $\sigma_{y}$ for a point $m$ in $M$, there exists a (positive) representing measure $\nu_{y}$ with $\nu_{y}$ absolutely continuous with respect to $\sigma_{y}$. Applying the Hoffman and Rossi theorem to $\sigma_{y}$, which is clearly absolutely continuous with respect to $\nu$, yields a representing measure $\nu_{y}$ for $y$ with

$$
\nu_{y}=h \mu+\epsilon \delta_{x} .
$$

It is now evident that $\nu_{y}^{\prime}=(h+\epsilon) \mu$ is the measure we seek. Thus, by Lemma $2.4, U \subset Q_{x}$ and $Q_{x}$ has positive $\lambda$-measure.

COROLlARY 3.4. There are at most countably many parts of $R(X)$ off the set of peak points of $R(X)$, each with positive $\lambda$-measure.

Corollary 3.5. A point of $X$ is a peak point of $R(X)$ if and only if $x$ is a point part (trivial part).

${ }^{2}$ Bishop has indicated how to avoid appealing to this result: If $\left\{f_{n}\right\} \subset A,\left\|f_{n}\right\| \leqq 1$ for all $n$ and $f_{n}(x) \rightarrow 1$, then $\int f_{n} d_{\mu \rightarrow 1}$. If $f$ is a weak* cluster point of $\left\{f_{n}\right\}$ in $L^{\infty}(\mu)$, then $f=1$ a.e. $-d_{\mu}$ and there is a subsequence $\left\{f_{n_{k}}\right\}$ such that $f_{n_{k}} \rightarrow f$ weak wence $^{*}$. Henctis $f_{n_{k}}(y)=\int f_{n_{k}} d \sigma_{y} \rightarrow 1$. This, by the definition of Gleason part, shows directly $y \in Q_{x}$. 
Proof. By the theorem point parts are peak points. But it is clear from the definition of peak point that such a point is always a point part.

CoRollary 3.6. If every point of $X$ is a point part, then $R(X)$ $=C(X)$.

Proof. By Corollary 3.5 this coincides with the well-known theorem of Bishop [2] for the minimal boundary of $R(X)$. (Hence Theorems 4 and 5 of [2] can be formulated in terms of point parts rather than the minimal boundary.)

It should be noted that the proof of Theorem 3.1 follows very closely the proof of Theorem 4 of [2] and that most of the work was already done by Bishop in that paper.

4. Concluding remarks. Apparently Theorem 3.1 is the first and only information available about the parts of $R(X)$ for an arbitrary compact set $X$ (For "nice" sets $X$ cf. [6] and [1].) However it hints at several other reasonable conjectures, the proofs of which seemingly should be as easily accessible as the above. Among these conjectures perhaps the foremost is the question of the connectivity of the parts. But also of interest is the question of whether two nontrivial parts may "touch" in the sense that points in one lie in the closure of another, or whether nontrivial parts must always be separated by peak points. These two questions are briefly summarized in the following conjecture, where $P$ denotes the set of peak points of $R(X)$.

Conjecture 4.1. Each component of $X-P$ is a nontrivial part of $R(X)$.

Although the number of nontrivial parts according to Corollary 3.4 is at most countable, and examples with an arbitrary finite number or countably many are trivial to construct, we have not been able to verify that, for a compact set without interior, $R(X)$ may have, say, just one nontrivial part. Since we feel this almost certainly must be possible, and can construct examples without interior in which a single part is dense, it would be interesting to see some such examples.

As a final remark we note that Corollary 3.6 provides a positive partial answer to the general conjecture that, for an arbitrary function algebra $A$ with maximal ideal space $M, A$ coincides with $C(M)$ if each point of $M$ is a point part.

\section{REFERENCES}

1. P. R. Ahern and Donald Sarason, On some hypo-Dirichlet algebras of analytic functions, Amer. J. Math. (to appear). 
2. E. Bishop, A minimal boundary for function algebras, Pacific J. Math. 9 (1959), 629-642.

3. - - Representing measures for points in a uniform algebra, Bull. Amer. Math. Soc. 70 (1964), 121-122.

4. A. M. Gleason, Function algebras, Seminar on analytic functions, Institute for Advanced Study, Princeton, N. J., 1957.

5. K. Hoffman and H. Rossi, On the extension of positive weak* continuous functionals, Duke Math. J. (to appear).

6. J. Wermer, Analytic disks in maximal ideal spaces, Amer. J. Math. 86 (1964), 161-170.

7. - Banach algebras and analytic functions, Advances in Math. 1 (1961), Fasc. 1, 51-102.

\section{BRANDEIS UNIVERSITY}

